\title{
Influence of the surface modification of alumina nanoparticles on the thermal stability and fire reaction of PMMA composites
}

\author{
Nicolas Cinausero', Nathalie Azema', Marianne Cochez ${ }^{2}$, Michel Ferriol ${ }^{2}$, \\ Mohamed Essahli ${ }^{3,4}$, François Ganachaud ${ }^{3}$ and José-Marie Lopez-Cuesta ${ }^{1 *}$ \\ ${ }^{1}$ Centre des Matériaux de Grande Diffusion, Ecole des Mines d'Alès, 6 avenue de Clavières, 30319 ALES Cedex, France \\ ${ }^{2}$ Département Chimie de l'IUT de Moselle-Est, Université Paul Verlaine-Metz, Laboratoire MOPS, UMR CNRS 7132, \\ 12 rue Victor Demange, 57500 Saint-Avold, France \\ ${ }^{3}$ Institut Charles Gerhardt, UMR5253 CNRS/UM2/ENSCM/UM1, Equipe Ingénierie et Architectures Macromoléculaires, \\ Ecole Nationale Supérieure de Chimie de Montpellier, 8 Rue de l'Ecole Normale, 34296 Montpellier cedex, France \\ ${ }^{4}$ Laboratoire des Procédés de Valorisation des ressources Naturelles, des Matériaux et Environnement, \\ Faculté des Sciences et Techniques de Settat, Km 3, route Casablanca, BP:577, 26000 Settat (Maroc), France
}

Nanometric aluminum oxide particles were modified by phosphonic acid-based oligomers of aromatic polyester, polyether, or polydimethylsiloxane (PDMS). The grafting process was charac-terized by FTIR spectroscopy and thermogravimetric analysis (TGA) showing that covalent bonds must have formed between the oxide and oligomers. The highest yield of grafting was achieved in dichloromethane $\left(\mathrm{CH}_{2} \mathrm{Cl}_{2}\right)$ solvent. Then, nanocomposites were prepared by melt-blending in a poly(methyl methacrylate) (PMMA) matrix. The best results in terms of thermal stability and flammability were obtained with the bis-phosphonicpolydimethylsiloxane-based formulation. With this latter, the peak of heat released rate (pHRR) decreased during the combustion, whereas PyGC/MS experiments led to the conclusion that PDMS-covered nanoparticles played a role in the composition of the gaseous phase as well.

KEYWORDS: nanocomposites; functionalization of polymers; flame retardancy

\section{INTRODUCTION}

Nanocomposites have drawn considerable attention over the last two decades. The incorporation of nanosized fillers in polymeric materials allows to enhance considerably a large range of properties. Among them, flammability appears to be improved by choosing nanoparticles as additives in flameretardant compositions. Their high specific surface area enables to engender many favorable interfacial interactions between the polymer matrix and fillers. Flame-retardancy of a wide variety of polymer layered silicate nanocomposites was studied and explained by barrier formation, ${ }^{1,2}$ catalytic action, and paramagnetic radical trapping. ${ }^{3,4}$ The differences observed for peak of heat released rate ( $p H R R$ ) of several polymers using cone calorimeter have been ascribed to polymer degradation pathway. ${ }^{4}$

Flame-retardancy of novel nanofillers as carbon nanotubes, ${ }^{5}$ layered double hydroxides, ${ }^{6}$ and also metal oxides ${ }^{7-10}$ was deeply studied in poly(methyl methacrylate) (PMMA).

*Correspondence to: J.-M. Lopez-Cuesta, Centre des Matériaux de Grande Diffusion, Ecole des Mines d'Alès, 6 avenue de Clavières 30319 ALES Cedex, France.

E-mail: jose-marie.lopez-cuesta@ema.fr
Laachachi et al. ${ }^{7}$ investigated the impact of $\mathrm{TiO}_{2}$ and $\mathrm{Fe}_{2} \mathrm{O}_{3}$ on thermal properties of PMMA nanocomposites and linked the increase of thermal stability $\left(+70^{\circ} \mathrm{C}\right)$ to the restriction of polymer chain mobility. Kashiwagi et al. ${ }^{8-10}$ explained that, in the condensed phase, the physical processes which significantly reduced pHRR were the accumulation of silica to the burning surface and its complete coverage. The fact that the nanosilica migrates toward the surface is not enough to provide an effective protective barrier; thanks to the formation of an in situ network, silica gel increases the effectiveness of flame-retardancy, however, without improving the thermal stability so much in this case.

The surface modification of oxide nanoparticles is a novel way for enhancing mechanical, ${ }^{11-12}$ optical, $^{13}$ and thermal ${ }^{14}$ properties of nanocomposites. Moreover, several possibilities of formation of hybrids between inorganic particles and organic compounds are available, according to literature:

- the "grafting onto" method which consists in making polymeric chains interact physically or chemically to inorganic particle surface ${ }^{15}$

- the "grafting from" method where polymerization is induced directly from oxide surface ${ }^{16}$ leading to inorganicorganic nanostructure 
- and the functionalization of the inorganic particles by coupling agents to ensure, for instance, high compatibility with a polymer matrix or to modify the hydrophobic/ hydrophilic balance of the nanoparticles. ${ }^{17}$

The present work concerns the modification of the aluminum oxide nanoparticle surface by phosphonic acidbased oligomers using a "grafting onto" method. The incorporation of the treated particles was carried out in PMMA and then, thermal/fire properties were evaluated and discussed. Specifically, the goal of the present work was to modify the interphase region of PMMA/alumina in order to achieve better thermal and fire properties and to investigate the degradation pathway of these nanocomposites.

\section{EXPERIMENTAL}

\section{Materials}

Pellets of PMMA (Altuglas V 825T, Arkema) were first reduced into powder in order to mix them physically with nanoparticles before being blended in an internal mixer. Commercial nanoalumina (Aeroxide AluC, Evonik Degussa), of $13 \mathrm{~nm}$ average particle size, was used as received (AluC) and also treated with organic compounds. Dichloromethane $\left(\mathrm{CH}_{2} \mathrm{Cl}_{2}\right)$ and tetrahydrofuran (THF) were purchased from Prolabo (France).

Bis-phosphonic acid compounds of polytetramethylene glycol ether (Terathane ${ }^{\circledR} 650$ or TER) and an aromatic polyester diol obtained through glycolysis of polyethylene terephtalate by diethylene glycol (PET) were prepared in two steps. ${ }^{18}$ Basically, an esterification by thioglycolic acid under acid conditions was followed by a radically controlled thiol-ene addition of vinylphosphonic acid. The purity of the intermediate and final bis-diphosphonic acid molecules were controlled by chain-end titrations and ${ }^{1} \mathrm{H}$ NMR.

Since PDMSs are sensitive to acid or base environment, a slightly different procedure has been chosen. In the first step, the reaction of esterification of the PDMS by the thioglycolic acid was carried out in the absence of the catalyst (dodecyl benzene sulphonic acid (DBSA)); the equilibrated reaction was simply moved toward the formation of the product by eliminating the water formed by drive azeotropic with toluene in Dean-Stark during $48 \mathrm{hr}$. In a second step, the radical addition of PDMS thiols onto vinyl phosphonate ester was performed under stoechiometric conditions. Finally, the cleavage of one ester unit per phosphonated groups was carried out by reaction with sodium iodide in anhydrous methylethylethyl cetone (MEEK) at reflux of solvent during $10 \mathrm{hr}$ followed by a protonation of the disalt in $1 \mathrm{M} \mathrm{HCl}$ water solution. ${ }^{19} \mathrm{H}$ and ${ }^{31} \mathrm{P}$ NMRs confirmed the monodealkylation (not shown).

In the following, bis-phosphonicpolydimethylsiloxane will designate the phosphonic acid derivated oligomer of PDMS, and so on. The chemical structures of the macromolecules are illustrated in Fig. 1.

\section{Grafting process onto the alumina nanoparticles}

Metallic oxide nanoparticles $(20 \mathrm{~g})$ AluC were dried $12 \mathrm{hr}$ at $110^{\circ} \mathrm{C}$ before being dispersed in the solvents (THF or $\mathrm{CH}_{2} \mathrm{Cl}_{2}$ ) with a concentration of $1 \mathrm{~g}$ per $50 \mathrm{ml}$. Meanwhile, the oligomers were solubilized in $40 \mathrm{ml}$ of the same solvent. The mass of the oligomers was relative to the calculated amount needed for the coverage of the oxide surface. The specific surface area of AluC was measured at $85.4 \mathrm{~m}^{2} \mathrm{~g}^{-1}$. The area occupied by the bis-phosphonic acid compounds was determined according to an estimation of the radius of gyration calculated from a randomcoil model: ${ }^{20}$

$$
R_{\mathrm{g}}=a N^{v}
$$

where $a$ is the Kuhn length estimated at $1.54 \AA, N$ the number of unit per molecule, and $v$ is an exponent equal to 0.6 that describes how $R_{\mathrm{g}}$ varies with the length of the oligomer. The estimated masses of PDMS, PET, and TER molecules are given in Table 1, from which five excesses were added to alumina for ensuring an efficient surface convering and coupling reaction. Then, the solution was added to the suspension of $\mathrm{AluC}$ and stirred for $24 \mathrm{hr}$ at room temperature (or with a reflux condenser at $40^{\circ} \mathrm{C}$ for $\mathrm{CH}_{2} \mathrm{Cl}_{2}$ ). Afterward, the mixture was centrifugated a first time. Then, after washing with the organic solvent and after another centrifugation, the collected solid phase was finally dried at $80^{\circ} \mathrm{C}$ for $6 \mathrm{hr}$ and then dried under vacuum at $80^{\circ} \mathrm{C}$ for $4 \mathrm{hr}$.<smiles>C=[Si](C)(C)O[Si](C)(C)O[Si](C)(C)C(C)(C)C(=O)CSCCP(=O)(O)OC(=O)CSCCP(=O)(O)O</smiles><smiles>O=C(COCCOC(=O)c1ccc(C(=O)OCCOCCOC(=O)c2ccc(OC(=O)CSCCP(=O)(O)O)cc2)cc1)OCCSCC(=O)OP(=O)(O)O</smiles>

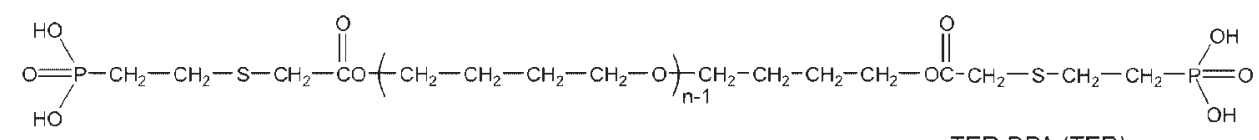

TER DPA (TER)

Figure 1. Bis-phosphonic acid oligomers based on polydimethylsiloxane (PDMS), polyethylene terephtalate diethylene glycol (PET) and Terathane ${ }^{\circledR}$ (TER). 
Table 1. Estimated mass of oligomers to graft on alumina oxide

\begin{tabular}{lccccc}
\hline & $\begin{array}{c}\text { Molar mass } \\
\left(\mathrm{g} \mathrm{mol}^{-1}\right)\end{array}$ & $\begin{array}{c}\text { Segment } \\
\text { number }\end{array}$ & $\begin{array}{c}\text { Radius of } \\
\text { giration }(\AA)\end{array}$ & $\begin{array}{c}\text { Covered } \\
\text { surface }(\AA)\end{array}$ & $\begin{array}{c}\text { Oligomer } \\
\text { mass } / \mathrm{g} \text { of AluC }(\mathrm{g})\end{array}$ \\
\hline PDMS & 1364 & 46 & 14.6 & 672 & 0.144 \\
PET & 854 & 35 & 12.4 & 488 & 0.124 \\
TER & 1014 & 59 & 16.9 & 901 & 0.080 \\
\hline
\end{tabular}

\section{Preparation of nanocomposites}

The test samples were prepared by mixing PMMA pellets and alumina fillers (each formulation containing $5 \mathrm{wt} \%$ of inorganic filler) in a Haake PolyLab internal mixer at $225^{\circ} \mathrm{C}$ and $50 \mathrm{rpm}$. The total mixing time was typically $8 \mathrm{~min}$. The resulting nanocomposites were crushed in a rotary cutter mill before being compression molded at $225^{\circ} \mathrm{C}$ for $5 \mathrm{~min}$ at 100 bars to obtain $100 \times 100 \times 4 \mathrm{~mm}^{3}$ specimens of around $50 \mathrm{~g}$ for cone calorimeter measurements. The same procedure was applied to pristine PMMA in order to compare it properly with filled compositions.

\section{Instrumentation}

Thermogravimetric analyses (TGA) were performed using a Perkin Elmer Pyris-1 TGA thermobalance operating under both air and nitrogen conditions in alumina crucibles containing around $10 \pm 2 \mathrm{mg}$ of material. The runs were carried out under dynamic conditions at the heating rate of

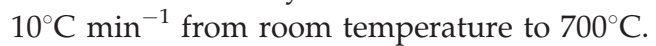

Glass transition temperatures $\left(T_{\mathrm{g}}\right)$ were determined by differential scanning calorimetry (DSC) using Pyris Diamond DSC Perkin Elmer apparatus with a heating rate of $10^{\circ} \mathrm{C} \mathrm{min}^{-1}$ between room temperature and $200^{\circ} \mathrm{C}$. Nitrogen was used as purge gas. $T_{\mathrm{g}}$ values are the average of two measurements.

ATR-FTIR spectra were recorded at room temperature on a Bruker IFS66 FTIR spectrometer (Golden Gate reflection system) to analyze the surface modification of nanoalumina. The spectra were measured with a spectral resolution of $2 \mathrm{~cm}^{-1}$.

Images of nanocomposites were obtained with a scanning transmission electron microscopy (STEM) detector coupled with a SEM microscope (FEI Quanta 200 SEM). All images were obtained under high vacuum at a voltage of $25.0 \mathrm{kV}$ with a spot size of 2.8 and a working distance of $8.2 \mathrm{~mm}$. X-ray microanalysis (EDX) performed with the same device was used to obtain information about powder surface.
Evaluation of the flammability properties of PMMA and its nanocomposites was made using a cone calorimeter device (Fire Testing Technology). Specimen sheets were exposed to a radiant cone under a heat flux of $35 \mathrm{~kW} \mathrm{~m}^{-2}$. Time to ignition (TTI), total heat release (THR), average and peak of heat released rate (HRR, Ave and pHRR) will be discussed. Results correspond to mean values obtained from two or three experiments.

PyGC/MS analyses were performed with a $6890 \mathrm{HP}$ Agilent chromatograph equipped with a CDS pyroprobe 2000 for flash pyrolysis. Flash pyrolysis was used during $60 \mathrm{sec}$ from 25 to $600^{\circ} \mathrm{C}$ (heating rate of $5000^{\circ} \mathrm{C} \mathrm{sec}^{-1}$ ). GC samples were injected in 20:1 split mode. GC oven was set to $100^{\circ} \mathrm{C}$ and equipped with a Permabond capillary column (cross linked 5\%PH-ME siloxane, $100 \mathrm{~m}$ length, $0.25 \mu \mathrm{m}$ film thickness). The GC was connected to a mass selective detector (HP Agilent 5973) operating in the electron impact mode at $70 \mathrm{eV}$ energy. As the carrier gas, helium was set at a constant flow of $40 \mathrm{ml} \mathrm{min}^{-1}$. Initial sample weight was $3 \pm 0.1 \mathrm{mg}$ for each experiment.

\section{RESULTS AND DISCUSSION}

\section{Grafting of oligomers onto alumina surface}

The phosphonic acid chain ends of each oligomer were chosen to ensure covalent bonds with the aluminum oxide nanoparticles. The possibility of modifying metallic oxide nanoparticles was successfully demonstrated by Guerrero et al. ${ }^{21}$ who modified nanoalumina by grafting phenylphosphonic acid and its organic-soluble ester derivatives. Thus, they verified the formation of Al-O-P leading to tridendate $\mathrm{PO}_{3}$ unit. Nevertheless a dissolutionprecipitation process may compete with surface modification according to severe experimental conditions. ${ }^{22}$ A schematic representation of the grafting process is illustrated in Fig. 2 involving the general structure of our oligomers with two potential active sites for coupling with the alumina nanoparticles.

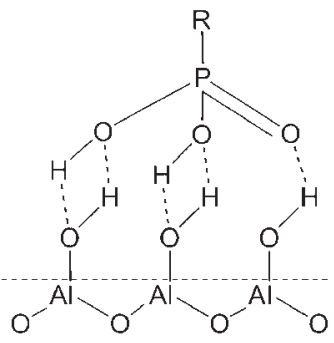

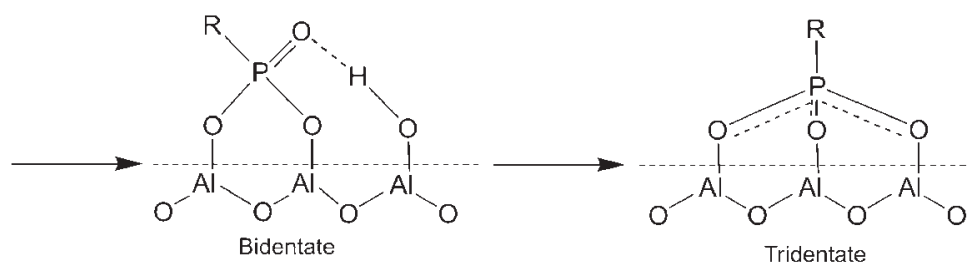

Figure 2. Schematization of the grafting process of phosphonic acid derivated compounds onto the oxide surface. 
Table 2. Alumina nanoparticles treated with phosphonic acid derivatives

\begin{tabular}{|c|c|c|c|c|c|}
\hline \multicolumn{2}{|c|}{ Powders and grafting conditions } & $\begin{array}{l}\text { Mass loss at } \\
700^{\circ} \mathrm{C}(\%)\end{array}$ & $\begin{array}{l}\text { Experimental organic/ } \\
\text { inorganic mass ratio (\%) }\end{array}$ & $\begin{array}{c}\text { Estimated organic/ } \\
\text { inorganic mass ratio (\%) }\end{array}$ & $S_{\mathrm{BET}}\left(\mathrm{m}^{2} \mathrm{~g}^{-1}\right)$ \\
\hline \multicolumn{2}{|l|}{ AluC } & 3.0 & 0.0 & - & 85.4 \\
\hline Alu-PDMS & THF $25^{\circ} \mathrm{C}$ & 8.7 & 5.7 & 12.6 & - \\
\hline Alu-PDMS & $\mathrm{CH}_{2} \mathrm{Cl}_{2} 25^{\circ} \mathrm{C}$ & 10.5 & 7.6 & 12.6 & 69.8 \\
\hline Alu-PDMS & $\mathrm{CH}_{2} \mathrm{Cl}_{2} 40^{\circ} \mathrm{C}$ & 10.5 & 7.5 & 12.6 & 68.0 \\
\hline Alu-PET & $\mathrm{CH}_{2} \mathrm{Cl}_{2} 25^{\circ} \mathrm{C}$ & 11.8 & 8.8 & 11.0 & 76.6 \\
\hline Alu-TER & $\mathrm{CH}_{2} \mathrm{Cl}_{2} 25^{\circ} \mathrm{C}$ & 12.7 & 9.8 & 7.4 & 61.0 \\
\hline
\end{tabular}

Mass loss was measured at $700^{\circ} \mathrm{C}$ by TGA analyses leading to the experimental ratio of organic compounds grafted onto the oxide.

TGA measurements of pristine and modified powders up to $700^{\circ} \mathrm{C}$ under air flow at $10^{\circ} \mathrm{C} \mathrm{min}^{-1}$ allowed the ratio of organic compound to be determined (Table 2). The weight loss of AluC at such a temperature revealed the dehydration and dehydroxylation reactions of oxide surface leading to a $3 \mathrm{wt} \%$ mass loss. The grafting conditions were optimized for Alu-PDMS. $\mathrm{CH}_{2} \mathrm{Cl}_{2}$ solvent favored a larger number of grafted macromolecules, but the temperature of reaction did not affect the organic/inorganic ratio. Comparing the estimated ratios toward experimental ones, we surprisingly obtained a higher yield of grafting than the estimated values except for TER. Furthermore, the decrease in specific surface area for treated aluminas could indicate the formation of an organic shell covering the surface of alumina particles or condensation processes between adjacent particles.

Figure 3 displays the DTG curves of Alu-PET and PET and Table 3 indicates the maximal degradation rate temperatures $\left(T_{\mathrm{dm}}\right)$ of the three types of modified alumina. $T_{\mathrm{dm}}$ of hybrids

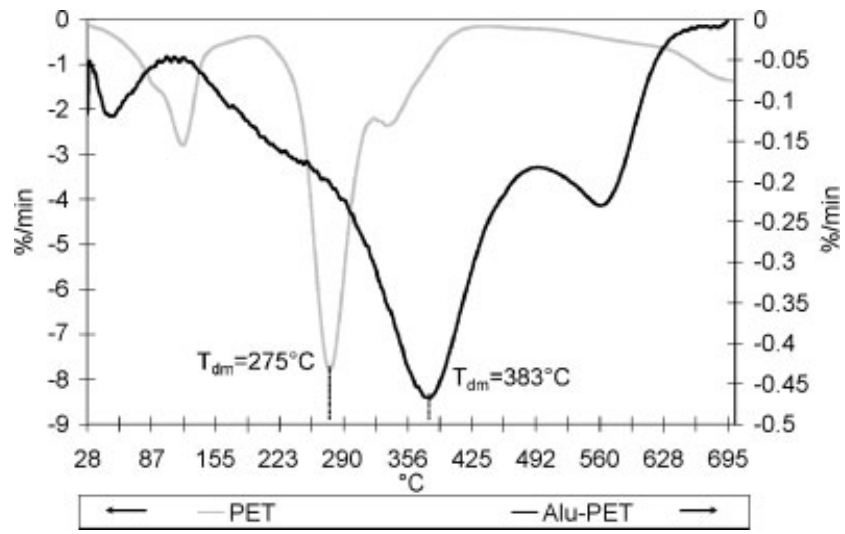

Figure 3. DTG curves of Alu-PET and PET at a heating rate of $10^{\circ} \mathrm{C} \mathrm{min}^{-1}$.

Table 3. Maximal degradation rate temperatures of oligomers and the corresponding modified particles, highly shifted to higher temperatures

\begin{tabular}{lc}
\hline & $T_{\mathrm{dm}}\left({ }^{\circ} \mathrm{C}\right)$ \\
\hline PDMS & 353 \\
Alu-PDMS & 378 \\
PETDEG & 275 \\
Alu-PET & 383 \\
TER & 247 \\
Alu-TER & 294 \\
\hline
\end{tabular}

particles are significantly shifted toward higher temperatures $\left(+108^{\circ} \mathrm{C}\right.$ for Alu-PET). This supposes that chemical interactions between the oxide and the oligomers could take place. The stabilization of the oligomers on the oxide surface rules out any possibility of decomposition during meltblending.

Figures 4-6 display the FTIR spectra of AluC, oligomers, and hybrid powders whereas IR absorption bands are detailed in Table 4. Since all the powders were dried before measurements, water or solvent residues could not be responsible for any kind of absorption. On the whole, the IR peaks of oligomers well match the peaks of hybrid particles which clearly indicate the success of grafting. In detail, the main IR band of AluC centered around $3400 \mathrm{~cm}^{-1}$ is the stretching signal of Al-OH hydroxyl groups. This signal is significantly decreased for hybrid alumina which shows an efficient coverage of the oxide surface by macromolecules with the assumption of covalent linkage. Figure 4 exhibits PDMS spectrum with several strong bands of polysiloxane units, at 1009 and $788 \mathrm{~cm}^{-1}$ belonging respectively to $\mathrm{Si}-\mathrm{O}$ and $\mathrm{Si}-\mathrm{CH}_{3}$. Such strong bands hide most of the organophosphorous vibrations whose wavenumber range is 1200-950 $\mathrm{cm}^{-1}$. Besides, comparing PDMS and Alu-PDMS, many frequencies are similar: $\mathrm{CH}_{2}$ vibrations $\left(2963 \mathrm{~cm}^{-1}\right.$ for Alu-PDMS), $\mathrm{C}=\mathrm{O}\left(1729 \mathrm{~cm}^{-1}\right)$.

Focusing on the $1300-900 \mathrm{~cm}^{-1}$ region of Figs. 5 and 6, it can be seen that vibrations of phosphonic acid groups $\mathrm{RP}(=$ O)-OH have two bands respectively at $1010-1000 \mathrm{~cm}^{-1}$ and $945 \mathrm{~cm}^{-1}$. The interesting point is the disappearance of these peaks for modified alumina reflecting well the modification

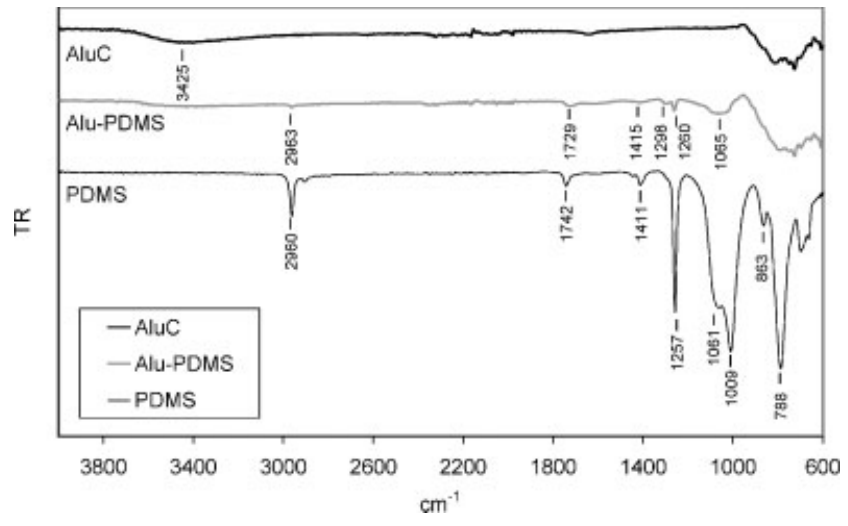

Figure 4. IR spectra of AluC and Alu-PDMS powders and PDMS oligomer. 


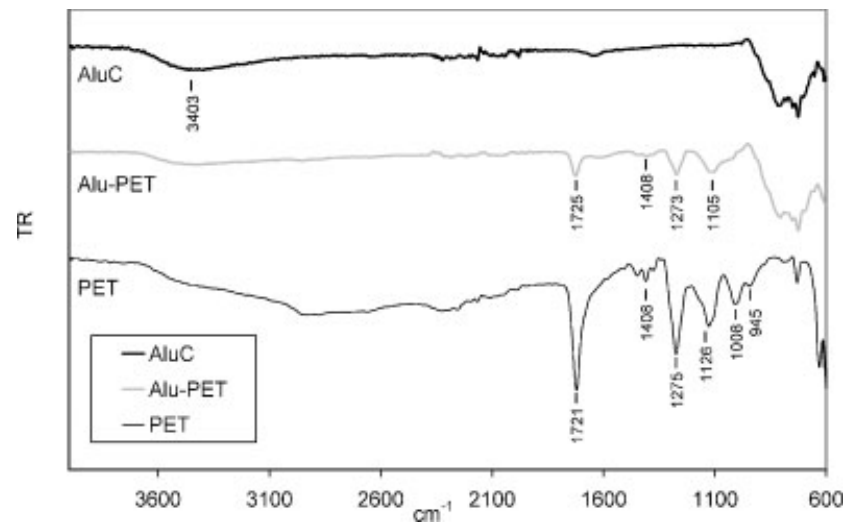

Figure 5. IR spectra of AluC and Alu-PET powders and PET oligomer.

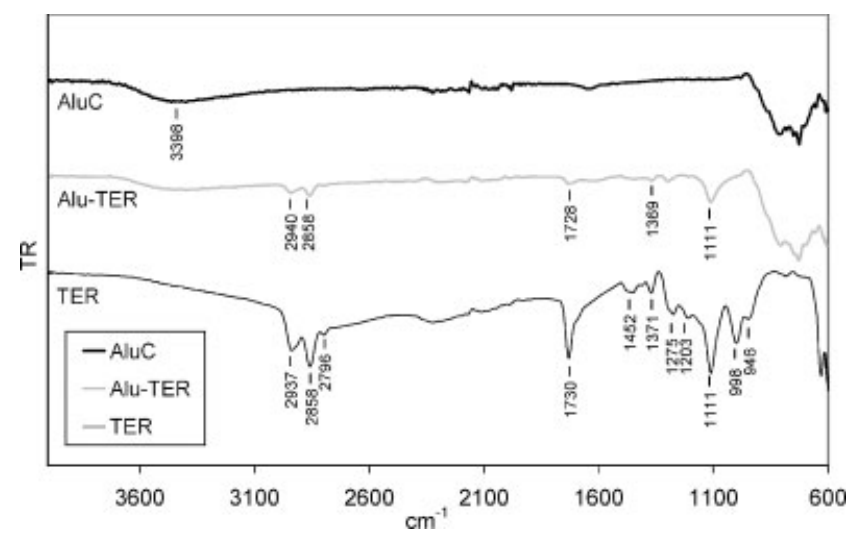

Figure 6. IR spectra of AluC and Alu-TER powders and TER oligomer.

of oligomers chain ends. Thus, this IR study demonstrates the modification of both alumina surface and oligomers chain ends which is in agreement with the formation of chemical bonds. Finally, the absence of $\mathrm{P}=\mathrm{O}$ vibrations in Alu-TER spectrum in Fig. 6 could indicate a tridentate coordination whereas in Fig. 5, Alu-PET IR spectrum exhibits this vibration at $1273 \mathrm{~cm}^{-1}$. Thus, the reaction with the oxide surface resulted in bidentate or tridentate coordination depends on the type of grafting molecule.

\section{Morphology of nanocomposites}

STEM micrographs of PMMA nanocomposites are presented in Fig. 7. The inorganic moieties are rather well dispersed and distributed in the polymeric matrix for any composition. The size range of particles is $10-200 \mathrm{~nm}$. The lower limit fits the primary particle size ( $13 \mathrm{~nm}$ for AluC). The morphology and size range of aggregates are similar for each composition. Even if the formation of bigger alumina aggregates could not be avoided, they are not displayed in the images since they are not representative of the general morphology of the nanocomposites. Such a dispersion was possible owing to both the proper shear rate reached in internal mixer and the good affinity between oxide and PMMA. Indeed, numerous interactions of the ester groups along the chain with oxide surface are ensured. ${ }^{23}$

\section{Thermal stability of nanocomposites}

TGA of PMMA and its nanocomposites are illustrated in Figs. 8 and 9 under air and nitrogen. The values of characteristic temperatures are detailed in Table 5. $T_{1 \%}$ and $T_{5 \%}$ are the temperatures corresponding respectively to 1 and $5 \%$ of weight loss.

First, gas-phase oxygen affects drastically the thermal degradation of PMMA $\left(-52^{\circ} \mathrm{C}\right.$ for $T_{5 \%}$ in comparison with nitrogen atmosphere). Under air, depolymerization of PMMA occurs at a lower temperature, since oxygen may favor random scission of macromolecules initiating unzipping of PMMA chain. ${ }^{24}$ The introduction of metallic oxide nanoparticles leads to a better thermal stability under air conditions as previously observed by others. ${ }^{25,26}$

The temperature of maximal degradation rate for AluC 5\% composition is almost the same under air and inert atmosphere (respectively $379^{\circ} \mathrm{C}$ and $383^{\circ} \mathrm{C}$ ) which means that the catalytic effect of oxygen observed in the thermal degradation of PMMA is suppressed by the presence of alumina. Physical interactions occur in the interfacial region of the nanocomposite avoiding the scissions at low temperature. ${ }^{23-26}$ Besides, chemical reactions may happen between ester side groups of PMMA and the hydroxyl groups of alumina creating carboxylate bonds. ${ }^{27,28}$ Therefore, all these interactions can annihilate early chain scissions caused by gas-phase oxygen effects. The restriction of chain backbone motions would be a consequence of these strong interactions.

Moreover, surface modification affects the thermal stability of the nanocomposites. Values between brackets

Table 4. IR absorption of the observed bands

\begin{tabular}{|c|c|c|c|}
\hline Bond & Vibration & Intensity & $\mathrm{cm}^{-1}$ \\
\hline $\mathrm{O}-\mathrm{H}$ & Stretching & Weak, wide & 3425 \\
\hline $\mathrm{CH}_{2}$ & Stretching & Medium & 2960 \\
\hline $\mathrm{C}=\mathrm{O}$ & Stretching & Strong & $1740-1720$ \\
\hline $\mathrm{P}-\mathrm{CH}_{2}-\mathrm{R}$ & Deformation & Medium to weak & 1420-1405 \\
\hline $\mathrm{Si}-\mathrm{CH}_{2}(-\mathrm{R})$ & Wagging & Medium & 1260 \\
\hline $\mathrm{P}=\mathrm{O}$ & Deformation & Strong & 1320-1200 \\
\hline $\mathrm{P}-\mathrm{O}$ & Stretching & Strong & $1200-1100$ \\
\hline$(\mathrm{Si}-\mathrm{O})_{n}$ & Stretching & Strong & 1100-1000 \\
\hline $\mathrm{RP}(=\mathrm{O})-\mathrm{OH}$ & Stretching & Medium & 1040-960, 950-900 \\
\hline $\mathrm{P}-\mathrm{C}$ & Stretching & Medium to weak & $780-620$ \\
\hline $\mathrm{Si}-\mathrm{CH}_{3}$, siloxane & Stretching/rocking & Strong & $830-740$ \\
\hline
\end{tabular}



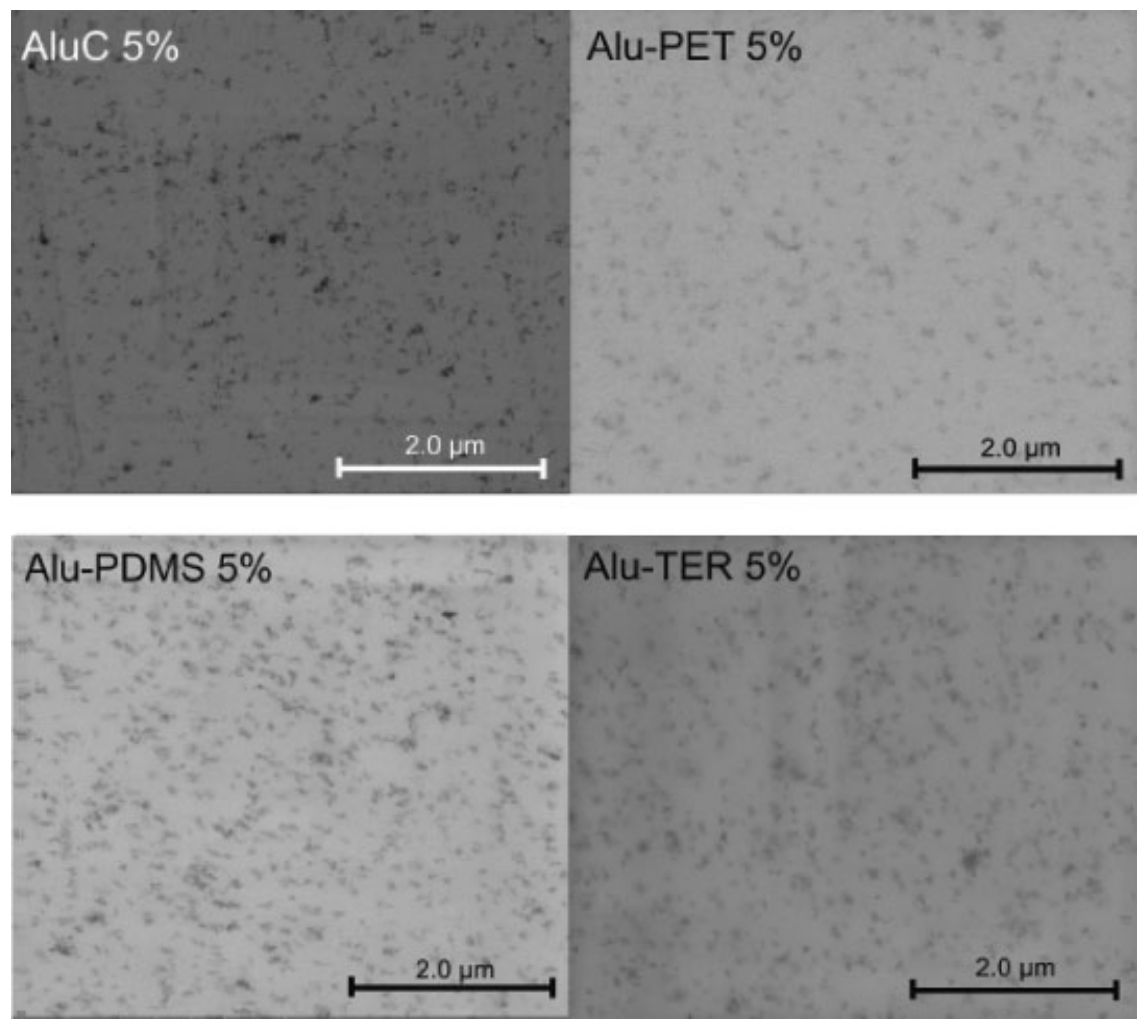

Figure 7. STEM micrographs of the PMMA nanocomposites loaded at $5 \mathrm{wt} \%$ of inorganic fillers.

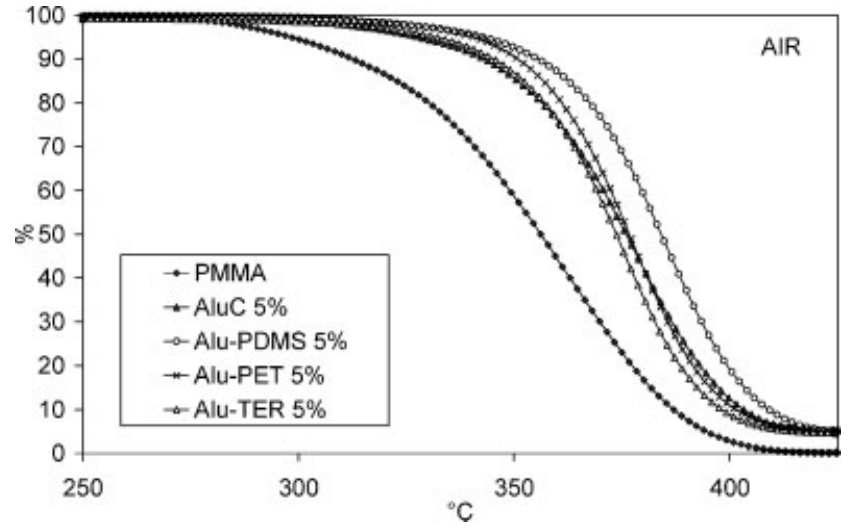

Figure 8. TGA curves of PMMA compositions with AluC and the treated alumina powders under air.

in Table 5 are the differences between characteristic temperatures of modified powders and temperatures of AluC compositions. These data give direct information on the stabilization or destabilization effect of the surface

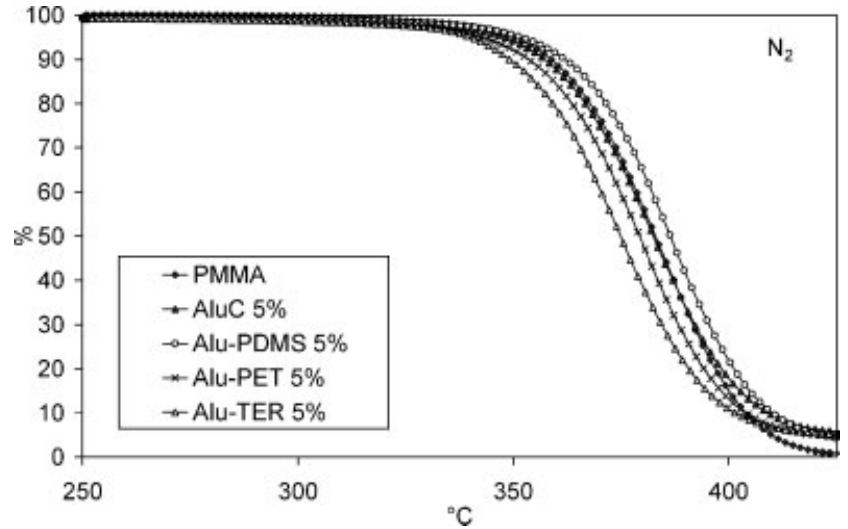

Figure 9. TGA curves of PMMA compositions with AluC and the treated alumina powders under nitrogen.

modification. Alu-PDMS filled nanocomposites are more stable in air atmosphere for any temperature than all AluC-based materials. The stability of nanocomposites in air follows the order of stability for grafted nanoparticles.

Table 5. Characteristic temperatures of degradation from TGA experiments

\begin{tabular}{|c|c|c|c|c|c|c|c|}
\hline & \multicolumn{3}{|c|}{ AIR } & \multicolumn{3}{|c|}{$\mathrm{N}_{2}$} & \multirow{2}{*}{$T_{\mathrm{g}}\left({ }^{\circ} \mathrm{C}\right)$} \\
\hline & $T_{1 \%}$ & $T_{5 \%}$ & $T_{\mathrm{dm}}$ & $T_{1 \%}$ & $T_{5 \%}$ & $T_{\mathrm{dm}}$ & \\
\hline PMMA & 276 & 298 & 362 & 320 & 350 & 385 & 116 \\
\hline AluC 5\% & 297 & 328 & 379 & 300 & 348 & 383 & 115 \\
\hline Alu-PDMS 5\% & $307\left(+10^{\circ} \mathrm{C}\right)$ & $343\left(+15^{\circ} \mathrm{C}\right)$ & $386\left(+7^{\circ} \mathrm{C}\right)$ & $308\left(+8^{\circ} \mathrm{C}\right)$ & $352\left(+4^{\circ} \mathrm{C}\right)$ & $387\left(+4^{\circ} \mathrm{C}\right)$ & 115 \\
\hline Alu-PET 5\% & $303\left(+6^{\circ} \mathrm{C}\right)$ & $341\left(+13^{\circ} \mathrm{C}\right)$ & $378\left(-1^{\circ} \mathrm{C}\right)$ & $284\left(-16^{\circ} \mathrm{C}\right)$ & $343\left(-5^{\circ} \mathrm{C}\right)$ & $380\left(-3^{\circ} \mathrm{C}\right)$ & 113 \\
\hline Alu-TER 5\% & $283\left(-14^{\circ} \mathrm{C}\right)$ & $332\left(+4^{\circ} \mathrm{C}\right)$ & $376\left(-3^{\circ} \mathrm{C}\right)$ & $279\left(-21^{\circ} \mathrm{C}\right)$ & $340\left(-8^{\circ} \mathrm{C}\right)$ & $375\left(-8^{\circ} \mathrm{C}\right)$ & 115 \\
\hline
\end{tabular}


Alu-PET and Alu-TER are destabilized under inert atmosphere. It is clear that the nature of the interphase region influences the degradation pathway of the polymer. Polyester and particularly polyether compounds tend to make easier the depolymerization of PMMA whereas the PDMS units, more thermally stable (as shown in Table 3), delay the unzipping of PMMA of $15^{\circ} \mathrm{C}$ at $5 \%$ weight loss compared to AluC-based formulation.

\section{Fire behavior and analysis of evolved products}

Figure 10 shows the evolution of HRR in function of time for the different compositions. All the corresponding data are presented in Table 6. The incorporation of AluC leads to a slight decrease of pHRR and HRR Ave value, as noticed in a previous work for a higher percentage of alumina $(15 \% \mathrm{wt}){ }^{29}$ This improvement of fire behavior has been interpreted in relation to both physical and physico-chemical processes, among which the modification of heat transfer through the material due to the presence of high specific surface area of particles was considered. Catalysis mechanisms ${ }^{28}$ can also modify the degradation pathway of filled PMMA, since charred residues are formed around the oxide particles. Grafting does not lead to dramatic modifications of fire reaction, particularly for THR values. Nevertheless, a further decrease of peak of HRR is noticed for Alu-PDMS

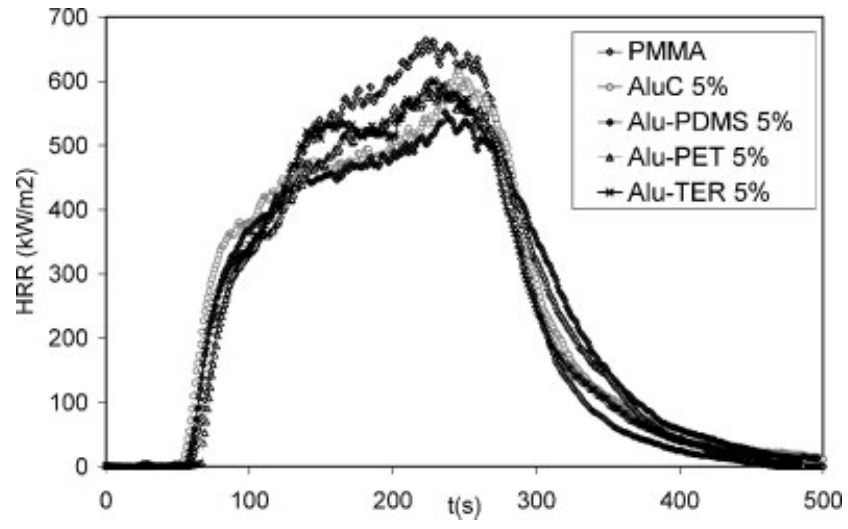

Figure 10. HRR curves of PMMA and PMMA compositions with $5 \%$ nanofillers.

composition in comparison with AluC one, owing to the stability conferred by the PDMS-based oligomer. One has to notice that this pHRR decrease is achieved using a very low amount of oligomer, corresponding to only $0.37 \%$ of the total weight of PMMA composite.

The increase in TSR can be ascribed to the specific degradation products of this latter. Conversely, the presence of Alu-TER entails a reduction for smoke opacity. Alu-PET

Table 6. Cone calorimeter data of PMMA compositions with $5 \%$ nanofillers

\begin{tabular}{|c|c|c|c|c|c|c|}
\hline & TTI (sec) & THR MJ m ${ }^{-2}$ & TSR $\mathrm{m}^{2} \mathrm{~m}^{-2}$ & HRR Ave $\mathrm{kW} \mathrm{m}^{-2}$ & pHRR $\mathrm{kW} \mathrm{m}^{-2}$ & Mass loss of chars at $800^{\circ} \mathrm{C}$ (ATG) \\
\hline PMMA & $57 \pm 5$ & $126 \pm 2$ & $479 \pm 44$ & $332 \pm 5$ & $639 \pm 7$ & - \\
\hline AluC 5\% & $52 \pm 3$ & $124 \pm 1$ & $456 \pm 39$ & $259 \pm 16$ & $601 \pm 15$ & 5.9 \\
\hline Alu PET 5\% & $67 \pm 2$ & $124 \pm 1$ & $441 \pm 30$ & $271 \pm 14$ & $593 \pm 2$ & 6.1 \\
\hline Alu TER 5\% & $58 \pm 2$ & $122 \pm 1$ & $363 \pm 53$ & $293 \pm 0$ & $600 \pm 3$ & 8.2 \\
\hline
\end{tabular}

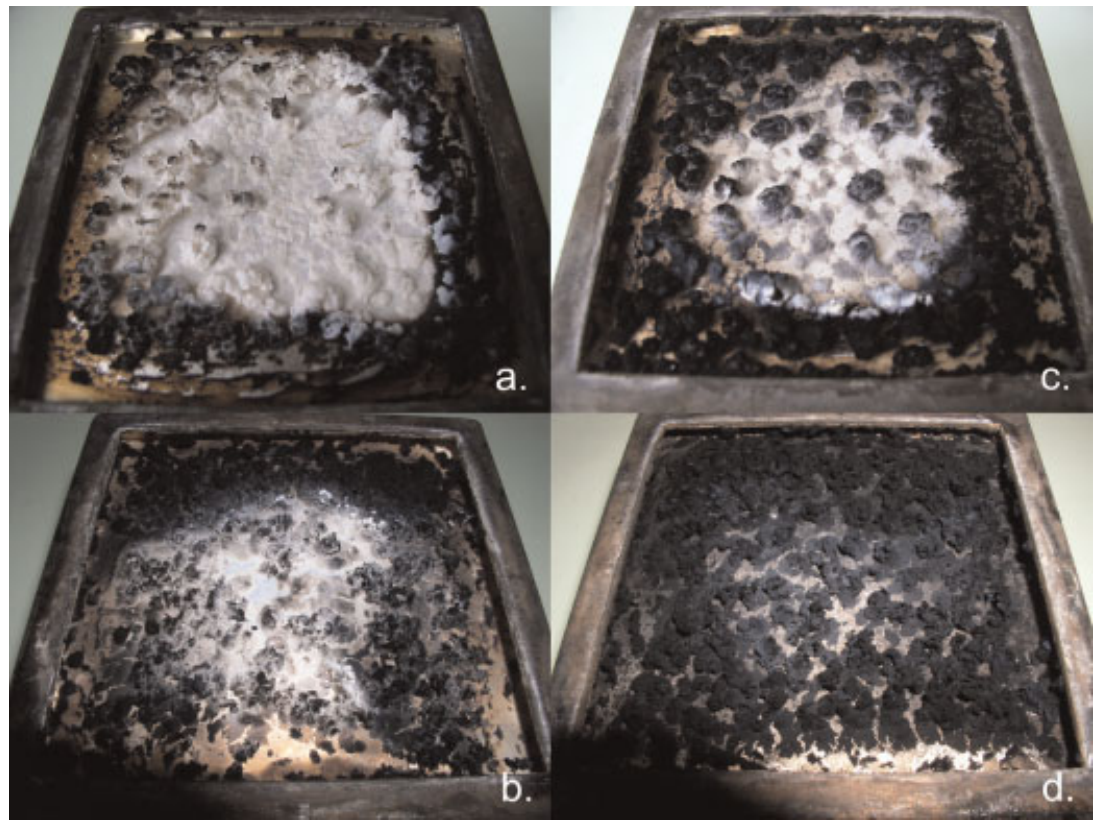

Figure 11. Photographs of char residues after cone calorimeter experiments from samples with PMMA 95\% and (a) AluC 5\%, (b) Alu-PDMS 5\%, (c) Alu-PET $5 \%$, (d) Alu-TER 5\%. This figure is available in color online at www.interscience. wiley.com/journal/pat 


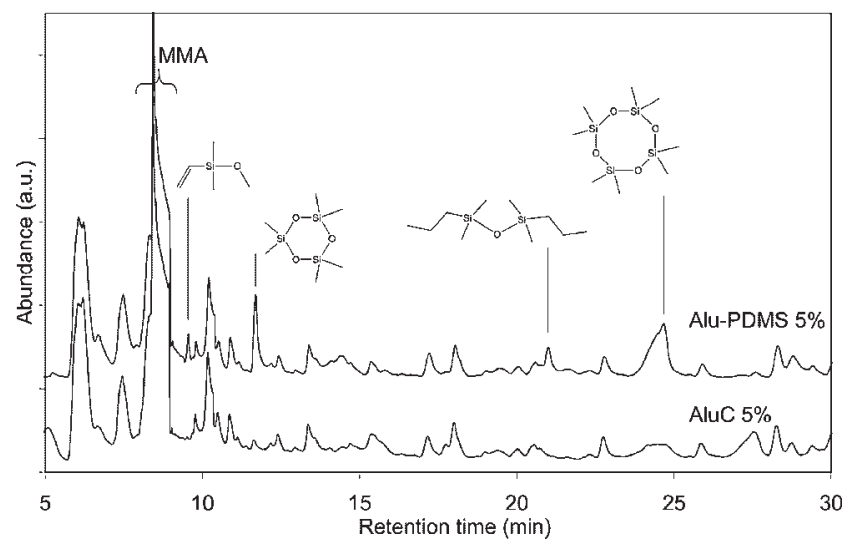

Figure 12. GC traces for the evolved product of PMMA composition with AluC and Alu-PDMS during thermal degradation.

composition tends to delay the TTI. To evaluate the amount of carbonaceous compounds in the residues, mass losses of cone calorimeter chars at $800^{\circ} \mathrm{C}$ have been determined by TGA (Table 6). It gives a quantitative indication of char promotion. AluC-based chars had the lowest mass loss whereas Alu-TER residues had the highest values. This observation seems to be confirmed by the pictures of residues displayed in Fig. 11. Modified particles led to higher rate of carbonaceous matter above all for Alu-PDMS and Alu-TER compositions. Comparing this two latter formulation, char promotion did not lead to better fire properties for Alu-TER composite which means that the improvement of Alu-PDMS fire properties could be caused by actions in gas phase as well.

In order to detail the behavior of Alu-PDMS composition, studies of AluC and Alu-PDMS compositions were carried out using PyGC-MS. The results are shown in Fig. 12. It revealed that the major degradation product was the MMA monomer as expected and that the two chromatograms were rather similar. The main differences are ascribed to different siloxane compounds (tricyclosiloxane in particular, as in the case of PDMS thermal degradation) in the case of Alu-PDMS which could account for the rise of smoke opacity. No compound containing sulfur and phosphorus could be detected indicating that they probably have a flame retardant action only in the condensed phase.

\section{CONCLUSION}

The modification of nanometric aluminum oxides particles by bis-phosphonic acid-based oligomers was achieved with the formation of covalent bonds between the oxide and the oligomers. The melt blending of the unmodified as well as the modified particles with PMMA led to well-dispersed nanocomposite structures. Thermal stability and fire behavior of PMMA were improved due to physical and physico-chemical processes involving the presence of the nanometric alumina. Significant improvements for these properties in relation to the grafting of the mineral were only noticed for the PDMS phosphonic acid-based formulation, despite the very low amount of oligomer present in PMMA. It is suggested that this compound could act in the condensed phase due to its important thermal stability and could also promote modifications of the degradation pathway of PMMA in the interphase region surrounding the alumina particles.

\section{REFERENCES}

1. Du J, Zhu J, Wilkie CA, Wang J. An XPS investigation of thermal degradation and charring on poly(vinyl chloride)clay nanocomposites. Polym. Degrad. Stab. 2002; 77: 377-381.

2. Laachachi A, Leroy E, Cochez M, Ferriol M, Lopez Cuesta JM. Use of oxide nanoparticles and organoclays to improve thermal stability and fire retardancy of PMMA. Polym. Degrad. Stab. 2005; 89: 344-352.

3. Zhu J, Uhl FM, Morgan AB, Wilkie CA. Studies on the mechanism by which the formation of nanocomposites enhances thermal stability. Chem. Mater. 2001: 13: 46494654.

4. Jang BN, Costache M, Wilkie CA. The relationship between thermal degradation behavior of polymer and the fire retardancy of polymer/clay nanocomposites. Polymer 2005; 46: 10678-10687.

5. Kashiwagi T, Du F, Winey KI, Grotha KM, Shields JR, Bellayer SP, Kim H, Douglas JF. Flammability properties of polymer nanocomposites with single walled nanotubes: effects of nanotube dispersion and concentration. Polymer 2005; 46: 471-481.

6. Costache MC, Wang D, Heidecker MJ, Manias E, Wilkie CA. The thermal degradation of PMMA nanocomposites with montmorillonite, layered double hydroxides and carbon nanotubes. Polym. Adv. Technol. 2006; 17: 272-280.

7. Laachachi A, Cochez M, Ferriol M, Lopez-Cuesta JM, Leroy E. Influence of $\mathrm{TiO}_{2}$ and $\mathrm{Fe}_{2} \mathrm{O}_{3}$ fillers on the thermal properties of PMMA. Mater. Lett. 2005; 59: 36-39.

8. Kashiwagi T, Morgan AB, Antonucci JM, VanLandingham MR, Harris RH, Awad WH, Shields JR. Thermal and flammability properties of a silica-PMMA nanocomposite. J. Appl. Polym. Sci. 2003: 89: 2072-2078.

9. Kashiwagi T, Gilman JW, Butler KM, Harris RH, Shields JR, Asano A. Flame retardant mechanism of silica gel/silica. Fire Mater. 2000; 24: 277-289.

10. Kashiwagi T, Shields JR, Harris RH, Davis RD. Flameretardant mechanism of silica: effect of resin molecular weight. J. Appl. Polym. Sci. 2003; 87: 1541-1553.

11. Bauer F, Decker U, Ernst $H$, Findeisen $M$, Langguth $H$, Mehnert R, Sauerland V, Hinterwaldner R. Functionalized inorganic/organic nanocomposites as new basic raw materials for adhesives and sealants Part 2. Int. J. Adhes. Adhesive. 2006; 26: 567-570.

12. Guo Z, Pereira T, Choi O, Wang Y, Hahn HT. Surface functionalized alumina nanoparticle filled polymeric materials. J. Mater. Chem. 2006; 16: 2800-2808.

13. Sugimoto H, Daimatsu K, Nakanishi E, Ogasawara Y, Yasumura T, Inomata K. Preparation and properties of poly (methylmethacrylate)-silica hybrid materials incorporating reactive silica nanoparticles. Polymer 2006; 47: 3754-3759.

14. Hong RY, Qian JZ, Cao JX. Synthesis and characterization of PMMA grafted ZnO nanoparticles. Powder Technol. 2006; 163: $160-168$.

15. Baskaran D, Mays JW, Bratcher MS. Polymer adsorption in the grafting reactions of hydroxyl terminal polymers with multi-walled carbon nanotubes. Polymer 2005; 46: 5050-5057.

16. Ninjbadgar T, Yamamoto $S$, Takano M. Thermal properties of the $\gamma-\mathrm{Fe}_{2} \mathrm{O}_{3} /$ poly(methyl methacrylate) core/shell nanoparticles. Solid State Sci. 2005; 7: 33-36.

17. Parida SK, Mishra PK, Mishra BK. Adsorption of styryl pyridinium dyes on CTAB treated silica. Indian J. Chem. 1999; 38A: 639-645.

18. Essahli M, Ganachaud F, In M, Boutevin B. Phosphonic acid functionalized polyethylene glycol and derivatives. J. Appl. Polym. Sci. 2008; 108: 483-490.

19. Koch P, Rumpel H, Sutter P, Weis CD. The reaction of alkanephosphonic acid esters with metals. Phosporus Sulfur Relat. Elem. 1989; 44: 75-85. 
20. Flory PJ. Statistical Mechanics of Chain Molecules Hanser/ Gardner Publications, Inc.: Cincinnati/Munich. 1988.

21. Guerrero G, Mutin HP, Vioux A. Organically modified aluminas by grafting and sol gel processes involving phosphonate derivatives. J. Mater. Chem. 2001; 11: 31613165.

22. Mutin HP, Guerrero G, Vioux A. Organic-inorganic hybrid materials based on organophosphorus coupling molecules: from metal phosphonates to surface modification of oxides. C. R. Chimie 2003; 6: 1153-1164.

23. Hamieh T, Schultz J. New approach to characterise physicochemical properties of solid substrates by inverse gas chromatography at infinite dilution: II. Study of the transition temperatures of poly(methyl methacrylate) at various tacticities and of poly(methyl methacrylate) adsorbed on alumina and silica. J. Chromatogr. A 2002; 969: 27-36.

24. Brown JE, Kashiwagi T. Gas phase oxygen on chain scission and monomer content in bulk PMMA degraded by external thermal radiation. Polym. Degrad. Stab. 1996; 52: $1-10$.

25. Hu Y-H, Chen C-Y, Wang C-C. Viscoelastic properties and thermal degradation kinetics of silica/PMMA nanocomposites. Polym. Degrad. Stab. 2004; 84: 545-553.

26. Yang F, Nelson GL. Polymer/silica nanocomposites prepared via extrusion. Polym. Adv. Technol. 2006; 17: 320-326.

27. Papirer E, Perrin JM, Nanse G, Fioux P. Adsorption of poly(methylmethacrylate) on an $\alpha$ alumina: evidence of formation of surface carboxylate bonds. Eur. Polym. J. 1994; 30: 985-991.

28. Liufu S-C, Xiao H-N, Li Y-P. Thermal analysis and degradation mechanism of polyacrylate/ $\mathrm{ZnO}$ nanocomposites. Polym. Degrad. Stab. 2005; 87: 103-110.

29. Laachachi A, Cochez M, Leroy E, Gaudon P, Ferriol M, Lopez Cuesta JM. Effect of $\mathrm{Al}_{2} \mathrm{O}_{3}$ and $\mathrm{TiO}_{2}$ nanoparticles and APP on thermal stability and flame retardance of PMMA. Polym. Adv. Technol. 2006; 17: 327-334. 